Pacific Journal of Mathematics

THE SHEAF OF OUTER FUNCTIONS IN THE POLYDISC 


\title{
THE SHEAF OF OUTER FUNCTIONS IN THE POLYDISC
}

\author{
Sergio E. Zarantonello
}

Let $U^{n}$ be the unit polydisc in $C^{n}$. Define a presheaf by assigning to each relatively open subset $W$ of $\bar{U}^{n}$ the multiplicative group of outer functions in the intersection $W \cap U^{n}$. If $\mathscr{Q}$ denotes the associated sheaf, we prove that $H^{q}\left(\bar{U}^{n}, \mathscr{Q}\right)=0$ for all integers $q \geqq 1$.

1. Introduction. Classically, the outer functions in the open unit disc $U$ are functions of the form

$$
\lambda \exp \int_{T} \frac{w+z}{w-z} k(w) d m(w),
$$

where $m$ is the Haar measure on the unit circle $T, k$ is an absolutely integrable real-valued function on $T$, and $\lambda$ is a complex number of modulus one. Closely related to the class of outer functions is the Smirnov class $N^{*}(U)$, which consists of all functions that are holomorphic in $U$ and admit an inner-outer factorization. The class $N^{*}(U)$ is an algebra, and the outer functions are precisely the invertible elements of this algebra. An alternative characterization of $N^{*}(U)$, considered by Rudin in [5], where it was extended to the polydisc $U^{n}$, is that a holomorphic function $f$ in $U$ belongs to $N^{*}(U)$ if and only if there exists a strongly convex function $\phi$ (depending on $f$ ) for which $\phi\left(\log ^{+}|f|\right)$ has a harmonic majorant. This definition can be extended naturally to arbitrary polydomains $W_{1} \times W_{2} \times \cdots \times W_{n}$, the requirement now being that $\phi\left(\log ^{+}|f|\right)$ have an $n$-harmonic majorant in $W_{1} \times W_{2} \times \cdots \times W_{n}$. We define the outer functions in $W_{1} \times W_{2} \times \cdots \times W_{n}$ to be the invertible elements of the algebra $N^{*}\left(W_{1} \times W_{2} \times \cdots \times W_{n}\right)$. (For the polydisc $U^{n}$, this definition can easily be seen to agree with the one given by Rudin in [5, Def. 4.4.3, p. 72].)

The correspondence that assigns to each polydomain $W$ in $\boldsymbol{C}^{n}$ the group $O\left(W \cap U^{n}\right)$ of outer functions in the intersection $W \cap U^{n}$, defines a sheaf $\mathscr{Q}$ on the closure $\bar{U}^{n}$ of $U^{n}$, which is locally determined in the sense that $\Gamma\left(\bar{U}^{n}, \mathbb{Q}\right)$ is canonically isomorphic to the group of outer functions in $U^{n}$. Our aim, in this article, is to show that the cohomology groups $H^{q}\left(\bar{U}^{n}, \mathscr{Q}\right)$ are trivial for all integers $q \geqq 1$.

Sheaves of a similar type (sheaves of germs of holomorphic functions satisfying boundary conditions on polydomains) have been studied by Nagel in [4], where a unified approach to many types of 
boundary behavior was given. Nagel's methods, however, do not appear to be applicable in our case. Instead, we use the methods developed by Stout in [7], which we also used in [8]. Indeed, the proof of Lemma 3.1 closely follows that of Lemma 1.2 of [7], and part of our conclusion is that the multiplicative Cousin problem with $N^{*}$-data can be solved in the polydisc.

II. Preliminaries. We denote the open unit disc $\{z \in C:|z|<1\}$ by $U$, and its boundary, the unit circle, by $T$. The cartesian product of $n$ copies of $U$ will be denoted by $U^{n}$. More generally, a polydomain in $\boldsymbol{C}^{n}$ will be a cartesian product $W_{1} \times W_{2} \times \cdots \times W_{n}$ of $n$ domains (open connected sets) in $C$. Similarly, $T^{n}$ will be the cartesian product of $n$ copies of $T$.

Let $W$ be an open set in $\boldsymbol{C}^{n}$, a continuous function $h: W \rightarrow$ $(-\infty,+\infty)$ is $n$-harmonic if it is harmonic in each complex variable separately; an upper semicontinuous function $s: W \rightarrow[-\infty,+\infty)$ is $n$-subharmonic if it is subharmonic in each complex variable separately. If $h$ and $s$ are as above, and if $s(z) \leqq h(z)$ for all $z \in W$, we say that $h$ is an $n$-harmonic majorant of $s$ in $W$.

The following proposition ([8, Th. 2.10, p. 301], see also [2]) shows that having an $n$-harmonic majorant is a local property under certain conditions.

Proposition 2.1. Let $W_{1}, W_{2}, \cdots, W_{n}$ be bounded domains in $C$ such that the boundary of each $W_{j}$ consists of finitely many mutually disjoint Jordan curves. Let $W=W_{1} \times W_{2} \times \cdots \times W_{n}$, and let $\left\{U_{\alpha}\right\}$ be a relatively open covering of the closure $\bar{W}$ of $W$. If $s$ is a positive n-subharmonic function in $W$ with "local" $n$ harmonic majorants $h_{\alpha}$ in each intersection $U_{\alpha} \cap W$, then $s$ must have an n-harmonic majorant in all of $W$.

Let $W$ be a polydomain in $C^{n}$. We define $N^{*}(W)$ to be the class of all holomorphic functions $f$ in $W$ such that $\phi\left(\log ^{+}|f|\right)$ has an $n$-harmonic majorant for some strongly convex function $\phi$. We recall that a function $\phi:(-\infty,+\infty) \rightarrow[0,+\infty)$ is strongly convex if it is convex, nondecreasing, and if $\lim _{t \rightarrow \infty} \phi(t) / t=+\infty$. Given two (or finitely many) strongly convex functions $\phi_{\alpha}$, it is always possible to find a strongly convex $\phi$ such that $\phi \leqq \phi_{\alpha}$ for all $\alpha$. This, together with the arithmetic properties of $\log ^{+}$, shows that $N^{*}(W)$ is closed under pointwise addition and multiplication, and is therefore an algebra. The class $O(W)$ of outer functions in $W$ is defined to be the group of all invertible elements of the algebra $N^{*}(W)$. If $W$ is simply connected, then $f \in O(W)$ if and only if $f=\exp g$, where $g=u+i v$ is holomorphic and where $\phi(|u|)$ has an $n$-harmonic ma- 
jorant in $W$ for some strongly convex function $\phi$. The additive group formed by such functions $g$ will be denoted $P(W)$.

Let $\Omega$ be the family of all cartesian products $W_{1} \times W_{2} \times \cdots \times W_{n}$, where each $W_{j}$ is connected and relatively open in $\bar{U}^{n}$. The pressheaves $W \rightarrow P\left(W \cap U^{n}\right), W \rightarrow O\left(W \cap U^{n}\right)$, defined for $W$ in $\Omega$, induce sheaves $\mathscr{P}$ and $\mathscr{Q}$ on $\bar{U}^{n}$. There is a canonical map $P\left(U^{n}\right) \rightarrow \Gamma\left(\bar{U}^{n}, \mathscr{P}\right)$ which is clearly one-one and a group homomorphism. To see that it is also onto, suppose $\left\{W_{\alpha}\right\}$ is a finite covering of $\bar{U}^{n}$ (by members of $\Omega$ ) and suppose that $f=u+i v$ is a holomorphic function in $U^{n}$ whose restriction to each intersection $W_{\alpha} \cap U^{n}$ is in $P\left(W_{\alpha} \cap U^{n}\right)$. For each $\alpha$ let $\phi_{\alpha}$ be a strongly convex function such that $\phi_{\alpha}(|u|)$ has an $n$-harmonic majorant in $W_{\alpha} \cap U^{n}$. Choose a strongly convex $\phi$ such that $\dot{\phi} \leqq \phi_{\alpha}$ for all $\alpha$. The $n$-subharmonic function $\phi(|u|)$ has $n$-harmonic majorants in the intersections $W_{\alpha} \cap U^{n}$. Consequently, by (2.1), it has an $n$-harmonic majorant in $U^{n}$. The function $f$ then belongs to $P\left(U^{n}\right)$, and the canonical map $P\left(U^{n}\right) \rightarrow \Gamma\left(\bar{U}^{n}, \mathscr{P}\right)$ is therefore an isomorphism. In a similar way we show that $O\left(U^{n}\right)$ and the group $\Gamma\left(\bar{U}^{n}, \mathbb{Q}\right)$ of global sections of $\mathscr{Q}$, are canonically isomorphic. More generally, if $W$ is a member of $\Omega, \Gamma(W, \mathscr{P})$ and $\Gamma(W, \mathscr{Q})$ can be naturally identified with the class of holomorphic functions in $W$ whose restriction to any $V \in \Omega$ such that $\bar{V} \subset W$, is in $P\left(V \cap U^{n}\right)$ and in $O\left(V \cap U^{n}\right)$ respectively.

In $\S I V$ we prove that $H^{q}\left(\bar{U}^{n}, \mathscr{Q}\right)=0$ for all integers $q \geqq 1$. First we need some technical results.

III. A generalized Cartan lemma. The following lemma is the crux of our work. It is a modified version of [7, Lemma 1.2, p. 380].

Let $\lambda_{1}$ and $\lambda_{2}$ be disjoint closed arcs on the circle $T$, and let $S^{2}$ be the extended complex plane. For $j=1,2$, define $V_{j}$ to be the union of the disc $U$, its exterior $S^{2}-\bar{U}$, and the interior (relative to $T$ ) of $\lambda_{j}$.

LEMma 3.1. If $f \in P\left(U^{n}\right)$, there exist functions $f_{j}$ which are holomorphic in $V_{j} \times U^{n-1}$, and such that:

(a) $f=f_{1}+f_{2}$ on $U^{n}$,

(b) $f_{j} \in P\left(U^{n}\right)$,

(c) $f_{j} \in P\left(\left(S^{2}-\bar{U}^{n}\right) \times U^{n-1}\right)$,

(d) $f_{j} \in P\left(D_{j} \times U^{n-1}\right)$, for some open disc $D_{j}$ containing $\lambda_{j}$.

Proof. We use the notation and terminology of [5]. In particular, $m_{n}$ will be the Haar measure on $T^{n}, Z^{n}$ will be the set of all $n$-tuples of integers, $Z_{+}^{n}$ the set of all $\alpha \in Z^{n}$ such that $\alpha_{1} \geqq 0, \alpha_{2} \geqq$ $0, \cdots, \alpha_{n} \geqq 0$, and $Y_{n}=Z_{+}^{n} \cup\left(-Z_{+}^{n}\right)$. For $z=\left(z_{1}, z_{2}, \cdots, z_{n}\right) \in U^{n}$ and 
$w=\left(w_{1}, w_{2}, \cdots, w_{n}\right) \in T^{n}, P_{n}\left(z_{1}, w\right)$ will be the $n$-dimensional Poisson kernel; i.e.,

$$
P_{n}(z, w)=\sum_{\alpha \in Z^{n}}|z|^{|\alpha|}\left(\frac{z \bar{w}}{|z|}\right)^{\alpha}
$$

where

$$
|\boldsymbol{z}|^{|\alpha|}=\left|\boldsymbol{z}_{1}\right|^{\left|\alpha_{1}\right|} \cdots\left|\boldsymbol{z}_{n}\right|^{\left|\alpha_{n}\right|} \text {, and }\left(\frac{z \bar{w}}{|\boldsymbol{z}|}\right)^{\alpha}=\left(\frac{z_{1} \bar{w}_{1}}{\left|z_{1}\right|}\right)^{\alpha_{1}} \cdots\left(\frac{z_{n} \bar{w}_{n}}{\left|z_{n}\right|}\right)^{\alpha_{n}} \text {. }
$$

We define $K_{n}(z, w)$ to be the summation in (1) restricted to the lattice points of $Y_{n}$. It can be verified that $K_{n}(z, w)$ is the real part of

$$
H_{n}(z, w)=\frac{2}{\left(1-\bar{w}_{1} z_{1}\right) \cdots\left(1-\bar{w}_{n} z_{n}\right)}-1 .
$$

In what follows, we will use an alternative characterization of $P\left(U^{n}\right)$. It is a consequence of [5, Th. 3.1.2, p. 37] and [5, Th. 3.2.4, p. 41] that a holomorphic function $f$ belongs to $P\left(U^{n}\right)$ if and only if its real part $u$ is the Poisson integral of some function $u^{*} \in$ $L^{1}\left(T^{n}\right)$; if this is the case, then $u^{*}(w)=\lim _{r \rightarrow 1^{-}} u\left(r w_{1}, r w_{2}, \cdots, r w_{n}\right)$ for almost all $w \in T^{n}$ (with respect to the measure $m_{n}$ ).

Suppose now that $f \in P\left(U^{n}\right)$, and write $f=f^{\prime}+f^{\prime \prime}$, where $f^{\prime}\left(z_{1}, z_{2}, \cdots, z_{n}\right)=f\left(0, z_{2}, \cdots, z_{n}\right)$. The function $f^{\prime}$ is clearly in $P\left(S^{2} \times U^{n-1}\right)$; therefore it suffices to prove the lemma for $f^{\prime \prime}$ instead of $f$. Let $u$ be the real part of $f^{\prime \prime}$ and let $u^{*}$ be the radial boundary values of $u$. Since the Fourier coefficients

$$
\widehat{u}^{*}(\alpha)=\int_{T^{n}} \bar{w}^{\alpha} u^{*}(w) d m_{n}(w)
$$

vanish for all $\alpha \notin Y_{n}$, we can write:

$$
u(z)=\int_{T^{n}} P_{n}(z, w) u^{*}(w) d m_{n}(w)=\int_{T^{\prime}} K_{n}(z, w) u^{*}(w) d m_{n}(w) .
$$

The kernel $H_{n}(z, w)$ is holomorphic in $z, H_{n}(0, w) \equiv 1$, and $K_{n}(z, w)=$ $\operatorname{Re} H_{n}(z, w)$. Therefore, since $f^{\prime \prime}(0)=0$, we have

$$
f^{\prime \prime}(z)=\int_{T^{n}} H_{n}(z, w) u^{*}(w) d m_{n}(w) .
$$

Choose an infinitely differentiable real-valued function $\chi$ on the circle $T$, such that $\chi$ is identically zero on an open connected subset $T_{1}$ of $T$ which contains $\lambda_{1}$, and identically one on a similar neighborhood $T_{2}$ of $\lambda_{2}$ in $T$. Define 


$$
\begin{aligned}
& f_{1}^{\prime \prime}(z)=\int_{T^{n}} H_{n}(z, w) \chi(w) u^{*}(w) d m_{n}(w), \\
& f_{2}^{\prime \prime}(z)=\int_{T^{n}} H_{n}(z, w)[1-\chi(w)] u^{*}(w) d m_{n}(w) .
\end{aligned}
$$

It is clear that $f_{j}^{\prime \prime}$ is not only holomorphic in $V_{j} \times U^{n-1}$ but at all points in $T_{j} \times U^{n-1}$ as well, and that $f_{1}^{\prime \prime}(z)+f_{2}^{\prime \prime}(z)=f^{\prime \prime}(z)$ for all $z \in U^{n}$.

We first prove that $f_{1}^{\prime \prime} \in P\left(U^{n}\right)$ (a symmetric argument will show that $\left.f_{2}^{\prime \prime} \in P\left(U^{n}\right)\right)$.

The function $\chi\left(w_{1}\right)$ is the sum of its Fourier series $\sum_{-\infty}^{+\infty} c_{k} w_{1}^{k}$, which converges uniformly and absolutely in $T$. Since $\chi$ is realvalued, we have $c_{-k}=\bar{c}_{k}$; also $\left|c_{k}\right|=O\left(k^{-q}\right)$ for all integers $q \geqq 1$.

If $u_{1}$ is the real part of $f_{1}^{\prime \prime}$, we have

$$
u_{1}(z)=\int_{T^{n}} K_{n}(z, w) \chi\left(w_{1}\right) u^{*}(w) d m_{n}(w) .
$$

To show that $f_{1}^{\prime \prime} \in P\left(U^{n}\right)$ it suffices to find a function $A \in L^{1}\left(T^{n}\right)$ such that

$$
\int_{T^{n}} K_{n}(z, w) \chi\left(w_{1}\right) u^{*}(w) d m_{n}(w)=\int_{T^{n}} P_{n}(z, w) A(w) d m_{n}(w) .
$$

This is trivially verified, with $A(w)=\chi\left(w_{1}\right) u^{*}(w)$, if $u\left(z_{1}, z_{2}, \cdots, z_{n}\right)$ depends only on $z_{1}$; for instance, if the radial boundary values of $u\left(z_{1}, 0, \cdots, 0\right)$ take the place of $u^{*}$. It therefore suffices to establish (3.1.1) with $u^{*}$ replaced by the radial boundary values of $u\left(z_{1}, z_{2}, \cdots, z_{n}\right)$ $u\left(z_{1}, 0, \cdots, 0\right)$. We assume then, without loss of generality, that $u\left(z_{1}, 0, \cdots, 0\right)$ is identically zero, or equivalently, that $\widehat{u}^{*}\left(\alpha_{1}, 0, \cdots, 0\right)=0$ for all integers $\alpha_{1}$. Write

$$
\begin{aligned}
u_{1}(z)= & c_{0} \int_{T^{n}} K_{n}(z, w) u^{*}(w) d m_{n}(w) \\
& +\sum_{k=1}^{\infty} \int_{T^{n}} K_{n}(z, w)\left[c_{k} w_{1}^{k}+\bar{c}_{k} \bar{w}_{1}^{k}\right] u^{*}(w) d m_{n}(w) .
\end{aligned}
$$

Let $\mu_{1}$ and $\nu_{1}$ be identically zero on $T^{n}$, and define, for $k=2,3, \cdots$, and for almost all $w \in T^{n}$,

$$
\begin{aligned}
& \mu_{k}(w)=\int_{T} u^{*}\left(w_{1} \bar{y}, w_{2}, \cdots, w_{n}\right) \sum_{j=1}^{k-1} \bar{y}^{j} d m_{1}(y), \\
& \nu_{k}(w)=\int_{T} u^{*}\left(w_{1} \bar{y}, w_{2}, \cdots, w_{n}\right) \sum_{j=1}^{k-1} \bar{y}^{j} d m_{1}(y),
\end{aligned}
$$

where $m_{1}$ is the Haar measure on the circle $T$. The functions $\mu_{k}, \nu_{k}$ belong to $L^{1}\left(T^{n}\right)$, and have $L^{1}$-norms no greater than $(k-1)\left\|u^{*}\right\|_{1}$. A simple calculation shows that the Fourier coefficients $\hat{\mu}_{k}\left(\alpha_{1}, \alpha_{2}, \cdots, \alpha_{n}\right)$ 
are zero unless $1-k \leqq \alpha_{1} \leqq-1$, in which case they agree with $\widehat{u}^{*}\left(\alpha_{1}, \alpha_{2}, \cdots, \alpha_{n}\right)$. We now recall that $f^{\prime \prime}\left(0, z_{2}, \cdots z_{n}\right) \equiv 0$, and consequently that the Fourier coefficients $\widehat{u}^{*}\left(0, \alpha_{2}, \cdots, \alpha_{n}\right)$ are all zero. This, together with the assumption that $\hat{u}^{*}\left(\alpha_{1}, 0, \cdots, 0\right)=0$ for all integers $\alpha_{1}$, and the series expansion of $K_{n}(z, w)$, shows (as in [7, p. 384]) that

$$
\begin{aligned}
& \int_{T^{n}} K_{n}(z, w) w_{1}^{k} u^{*}(w) d m_{n}(w) \\
& \quad=\int_{T^{n}} P_{n}(z, w) w_{1}^{k}\left[u^{*}(w)-\mu_{k}(w)\right] d m_{n}(w) .
\end{aligned}
$$

Similarly

$$
\begin{aligned}
& \int_{T^{n}} K_{n}(z, w) \bar{w}_{1}^{k} u^{*}(w) d m_{n}(w) \\
& \quad=\int_{T^{n}} P_{n}(z, w) \bar{w}_{1}^{k}\left[u^{*}(w)-\nu_{k}(w)\right] d m_{n}(w) .
\end{aligned}
$$

If we define $A_{k}(w)=c_{k} w_{1}^{k}\left[u^{*}(w)-\mu_{k}(w)\right]+\bar{c}_{k} \bar{w}_{1}^{k}\left[u^{*}(w)-\nu_{k}(w)\right]$, and combine (3.1.3) and (3.1.4), we have

$$
\begin{gathered}
\int_{T^{n}} K_{n}(z, w)\left[c_{k} w_{1}^{k}+\bar{c}_{k} \bar{w}_{1}^{k}\right] u^{*}(w) d m_{n}(w) \\
=\int_{T^{n}} P_{n}(z, w) A_{k}(w) d m_{n}(w) .
\end{gathered}
$$

The estimates $\left\|\mu_{k}\right\|_{1} \leqq(k-1)\left\|u^{*}\right\|_{1},\left\|\nu_{k}\right\|_{3} \leqq(k-1)\left\|u^{*}\right\|_{1}$, show that $\left\|A_{k}\right\|_{1} \leqq 2\left\|u^{*}\right\|_{1}\left|c_{k}\right| k$. Since $\sum_{k=1}^{\infty}\left|c_{k}\right| k$ converges, the series $\sum_{k=1}^{\infty} A_{k}$ converges absolutely in $L^{1}\left(T^{n}\right)$. If $A=c_{0} u^{*}+\sum_{k=1}^{\infty} A_{k}$, (3.1.2) and (3.1.5) show that (3.1.1) is verified. Consequently $f_{1}^{\prime \prime} \in P\left(U^{n}\right)$.

Next we prove that $f_{1}^{\prime \prime}$ and $f_{2}^{\prime \prime}$ are in $P\left(\left(S^{2}-\bar{U}^{n}\right) \times U^{n-1}\right)$.

A direct calculation yields

$$
\begin{aligned}
& f_{1}^{\prime \prime}\left(z_{1}, z_{2}, \cdots, z_{n}\right)-f_{1}^{\prime \prime}\left(\frac{1}{\bar{z}_{1}}, z_{2}, \cdots, z_{n}\right) \\
& \quad=\int_{T^{n}} P_{1}\left(z_{1}, w_{1}\right) \frac{2}{\left(1-z_{2} \bar{w}_{2}\right) \cdots\left(1-z_{n} \bar{w}_{n}\right)} \chi\left(w_{1}\right) u^{*}(w) d m_{n}(w)
\end{aligned}
$$

for all $z \in U^{n}$. (Here, $P_{1}\left(z_{1}, w_{1}\right)$ is the one-dimensional Poisson kernel.) Taking real parts in (3.1.6), we get

$$
\begin{aligned}
u_{1}\left(z_{1}, z^{\prime}\right)-u_{1}\left(\frac{1}{\bar{z}_{1}}, z^{\prime}\right) \\
=\int_{T n} P_{1}\left(z_{1}, w_{1}\right)\left[1+K_{n-1}\left(z^{\prime}, w^{\prime}\right)\right] \chi\left(w_{1}\right) u^{*}(w) d m_{n}(w) \\
=\int_{T n} P_{1}\left(z_{1}, w_{1}\right) \chi\left(w_{1}\right) u^{*}(w) d m_{n}(w) \\
\quad+\int_{T^{n}} P_{1}\left(z_{1}, w_{1}\right) K_{n-1}\left(z^{\prime}, w^{\prime}\right) \chi\left(w_{1}\right) u^{*}(w) d m_{n}(w)
\end{aligned}
$$


where $z^{\prime}=\left(z_{2}, \cdots, z_{n}\right)$ and $w^{\prime}=\left(w_{2}, \cdots, w_{n}\right)$.

Since the Fourier coefficients of $\chi\left(w_{1}\right) u^{*}(w)=\sum_{-\infty}^{+\infty} c_{k} w_{1}^{k} u^{*}(w)$ are zero for all lattice points not in $Z \times Y_{n-1}$, and since

$$
P_{1}\left(z_{1}, w_{1}\right) K_{n-1}\left(z^{\prime}, w^{\prime}\right)=\sum_{\alpha \in Z \times Y}|z|^{|\alpha|}\left(\frac{z \bar{w}}{|z|}\right)^{\alpha},
$$

we can write

$$
\begin{gathered}
\int_{T^{n}} P_{1}\left(z_{1}, w_{1}\right) K_{n-1}\left(z^{\prime}, w^{\prime}\right) \chi\left(w_{1}\right) u^{*}(w) d m_{n}(w) \\
=\int_{T^{n}} P_{n}(z, w) \chi\left(w_{1}\right) u^{*}(w) d m_{n}(w) .
\end{gathered}
$$

On the other hand, if we define

$$
v^{*}\left(w_{1}\right)=\int_{T^{n-1}} u^{*}\left(w_{1}, w^{\prime}\right) d m_{n-1}\left(w^{\prime}\right),
$$

then

$$
\begin{aligned}
& \int_{T^{n}} P_{1}\left(z_{1}, w_{1}\right) \chi\left(w_{1}\right) u^{*}(w) d m_{n}(w) \\
& \quad=\int_{T} P_{1}\left(z_{1}, w_{1}\right) \chi\left(w_{1}\right) v^{*}\left(w_{1}\right) d m_{1}\left(w_{1}\right) \\
& \quad=\int_{T^{n}} P_{n}(z, w) \chi\left(w_{1}\right) v^{*}\left(w_{1}\right) d m_{n}(w) .
\end{aligned}
$$

Substituting (3.1.8) and (3.1.9) in (3.1.7) yields

$$
u_{1}\left(z_{1}, z^{\prime}\right)-u_{1}\left(\frac{1}{\bar{z}_{1}}, z^{\prime}\right)=\int_{T^{n}} P_{n}(z, w)\left[\chi\left(w_{1}\right) v^{*}\left(w_{1}\right)+\chi\left(w_{1}\right) u^{*}(w)\right] d m_{n}(w),
$$

which allows us to write

$$
u_{1}\left(\frac{1}{\bar{z}_{1}}, z^{\prime}\right)=\int_{T^{n}} P_{n}(z, w)\left[u_{1}^{*}(w)-\chi\left(w_{1}\right) v^{*}\left(w_{1}\right)-\chi\left(w_{1}\right) u^{*}(w)\right] d m_{n}(w) .
$$

The above exhibits $u_{1}\left(1 / \bar{z}_{1}, z^{\prime}\right)$ as the Poisson integral of a function in $L^{1}\left(T^{n}\right)$. This implies ([5, Th. 3.2.4, p. 41]) that there exists a strongly convex $\phi$ and an $n$-harmonic function $h$ in $U^{n}$ such that

$$
\phi\left(\left|u_{1}\left(\frac{1}{\bar{z}_{1}}, z^{\prime}\right)\right|\right) \leqq h\left(z_{1}, z^{\prime}\right)
$$

for all $\left(z_{1}, z^{\prime}\right) \in U^{n}$. Consequently

$$
\phi\left(\left|u_{1}(z)\right|\right) \leqq h\left(\frac{1}{\bar{z}_{1}}, z^{\prime}\right),
$$

for $z \in\left(S^{2}-\bar{U}\right) \times U^{n-1}$. Since $h\left(1 / \bar{z}_{1}, z^{\prime}\right)$ is $n$-harmonic in $\left(S^{2}-\bar{U}\right) \times$ $U^{n-1}, f_{1}^{\prime \prime}$ must belong to $P\left(\left(S^{2}-\bar{U}\right) \times U^{n-1}\right)$. Similarly, we show 
that $f_{2}^{\prime \prime} \in P\left(\left(S^{2}-\bar{U}\right) \times U^{n-1}\right)$.

Finally, we prove part (d) of the lemma.

Denote by $h_{U}$ and $h_{S^{2}-\bar{U}}$ the least $n$-harmonic majorants of $\left|u_{1}\right|$ in $U^{n}$ and $\left(S^{2}-\bar{U}\right) \times U^{n-1}$ respectively. (That $h_{U}$ and $h_{S^{2}-\bar{U}}$ exist is a direct consequence of parts (b) and (c) of the lemma.) As functions of the single variable $z_{1}, h_{U}\left(z_{1}, 0, \cdots, 0\right)$ and $h_{S^{2}-\bar{U}}\left(z_{1}, 0, \cdots, 0\right)$ are positive harmonic functions (in $U$, and in $S^{2}-\bar{U}$ ). Therefore, as is well known, they must have nontangential boundary values at almost all points of $T$. Choose in each of the two connected components of $T_{1}-\lambda_{1}$ a point where both $h_{U}\left(z_{1}, 0, \cdots, 0\right)$ and $h_{s^{2}-\bar{U}}\left(z_{1}, 0, \cdots, 0\right)$ simultaneously have a nontangential boundary value. Call these points $\zeta^{\prime}$ and $\zeta^{\prime \prime}$, and let $C$ be a circle which intersects the circle $T$ precisely at $\zeta^{\prime}$ and $\zeta^{\prime \prime}$. If $C$ has center $a$ and radius $\rho$ we write $C=a+\rho T$. Let $D_{1}$ be the disc bounded by $a+\rho T$, and let $W_{1}=$ $U \cup T_{1} \cup\left(S^{2}-\bar{U}\right)$. As we mentioned earlier, $f_{1}^{\prime \prime}$ is holomorphic in $W_{1} \times U^{n-1}$. Thus, for each $z^{\prime}=\left(z_{2}, \cdots, z_{n}\right) \in U^{n-1}$, the function $z_{1} \rightarrow$ $f_{1}^{\prime \prime}\left(z_{1}, z^{\prime}\right)$ is holomorphic in $W_{1}$. Since the closure of $D_{1}$ is contained in $W_{1}$, the function $u_{1}\left(z_{1}, z^{\prime}\right)$ can be represented there as the Poisson integral of its values on the circle $a+\rho T$, i.e.,

$$
u_{1}\left(z_{1}, z^{\prime}\right)=\int_{T} u_{1}\left(a+\rho w_{1}, z^{\prime}\right) P_{1}\left(\frac{z_{1}-a}{\rho}, w_{1}\right) d m_{1}\left(w_{1}\right),
$$

for all $z=\left(z_{1}, z^{\prime}\right) \in D_{1} \times U^{n-1}$.

Similarly, for each $z_{1}$ in $U$ or in $S^{2}-\bar{U}$, the function $z^{\prime} \rightarrow$ $f_{1}^{\prime \prime}\left(z_{1}, z^{\prime}\right)$ is holomorphic in $U^{n-1}$, and belongs to $P\left(U^{n-1}\right)$ by parts $(\mathrm{b})$ and (c) of the lemma. Thus $z^{\prime} \rightarrow u_{1}\left(z_{1}, z^{\prime}\right)$ has radial boundary values $u_{1}\left(z_{1}, w^{\prime}\right)$ in $L^{1}\left(T^{n-1}\right)$, and

$$
u_{1}\left(z_{1}, z^{\prime}\right)=\int_{T^{n-1}} u_{1}\left(z_{1}, w^{\prime}\right) P_{n-1}\left(z^{\prime}, w^{\prime}\right) d m_{n-1}\left(w^{\prime}\right),
$$

for all $z=\left(z_{1}, z^{\prime}\right)$ either in $U^{n}$ or in $\left(S^{2}-\bar{U}\right) \times U^{n-1}$.

A point $a+\rho w_{1}$ on the circle $a+\rho T$ will be contained in $U$ or in $S^{2}-\bar{U}$, or will be one of the two intersections $\zeta^{\prime}$ and $\zeta^{\prime \prime}$ of $a+\rho T$ with $T$. In the first two cases, by (3.1.11), we have

$$
u_{1}\left(\alpha+\rho w_{1}, z^{\prime}\right)=\int_{T^{n-1}} u_{1}\left(\alpha+\rho w_{1}, w^{\prime}\right) P_{n-1}\left(z^{\prime}, w^{\prime}\right) d m_{n-1}\left(w^{\prime}\right) .
$$

Substituting the above in (3.1.10), we obtain

$$
\begin{aligned}
u_{1}\left(z_{1}, z^{\prime}\right)=\int_{T} & \left\{\int_{T^{n-1}} u_{1}\left(\alpha+\rho w_{1}, w^{\prime}\right) P_{n-1}\left(z^{\prime}, w^{\prime}\right) d m_{n-1}\left(w^{\prime}\right)\right\} \\
& P_{1}\left(\frac{z_{1}-a}{\rho}, w_{1}\right) d m_{1}\left(w_{1}\right),
\end{aligned}
$$


for all $z=\left(z_{1}, z^{\prime}\right) \in D_{1} \times U^{n-1}$.

The function $u_{1}\left(a+\rho w_{1}, w^{\prime}\right)$ is measurable on $T^{n}$, and for each $w_{1} \in T$ belongs (as a function of $w^{\prime}$ ) to $L^{1}\left(T^{n}\right)$. We next show that $u_{1}\left(\alpha+\rho w_{1}, w^{\prime}\right)$ is in $L^{1}\left(T^{n}\right)$.

For a fixed point $a+\rho w_{1}$ in $a+\rho T$, the function

$$
I\left(\alpha+\rho w_{1}, z^{\prime}\right)=\int_{T^{n-1}}\left|u_{1}\left(\alpha+\rho w_{1}, w^{\prime}\right)\right| P_{n-1}\left(z^{\prime}, w^{\prime}\right) d m_{n-1}\left(w^{\prime}\right)
$$

is the least $n-1$-harmonic majorant of $z^{\prime} \rightarrow\left|u_{1}\left(a+\rho w_{1}, z^{\prime}\right)\right|$ in $U^{n-1}$. Since $\left|u_{1}\right|$ has $n$-harmonic majorants $h_{U}$ and $h_{S^{2}-\bar{U}}$ in $U_{n}$ and $S^{2}-\bar{U}$ respectively, we have the inequalities

$$
I\left(a+\rho w_{1}, z^{\prime}\right) \leqq h_{U}\left(\alpha+\rho w_{1}, z^{\prime}\right), \quad \text { if } \quad a+\rho w_{1} \in U,
$$

and

$$
I\left(a+\rho w_{1}, z^{\prime}\right) \leqq h_{S^{2}-\bar{U}}\left(a+\rho w_{1}, z^{\prime}\right), \quad \text { if } \quad a+\rho w_{1} \in S^{2}-\bar{U} .
$$

Recalling that $h_{U}\left(z_{1}, 0\right)$ has limits as $z_{1}$ approaches $\zeta^{\prime}$ and $\zeta^{\prime \prime}$ nontangentially, it follows that $h_{U}\left(z_{1}, 0\right)$ is bounded on the intersection $a+\rho T \cap U$ (since the circle $a+\rho T$ meets $T$ nontangentially at $\zeta^{\prime}$ and $\left.\zeta^{\prime \prime}\right)$. Similarly, $h_{S^{2}-\bar{v}}\left(z_{1}, 0\right)$ is bounded on $a+\rho T \cap S^{2}-\bar{U}$. Thus there exists a constant $M$ such that $h_{U}\left(a+\rho w_{1}, 0\right) \leqq M$ if $a+\rho w_{1} \in U$, and $h_{S^{2}-\bar{U}}\left(a+\rho w_{1}, 0\right) \leqq M$ if $a+\rho w_{1} \in S^{2}-\bar{U}$. Therefore, if we let $z^{\prime}=0$ in (3.1.13), we get

$$
\left.\int_{T^{n-1}} \mid u_{1}\left(\alpha+\rho w_{1}\right) w^{\prime}\right) \mid d m_{n-1}\left(w^{\prime}\right) \leqq M,
$$

for all $w_{1} \in T$. Hence

$$
\int_{T} \int_{T^{n-1}}\left|u_{1}\left(a+\rho w_{1}, w^{\prime}\right)\right| d m_{n-1}\left(w^{\prime}\right) d m_{1}\left(w_{1}\right) \leqq M,
$$

which shows that $u_{1}\left(\alpha+\rho w_{1}, w^{\prime}\right)$ is in $L^{1}\left(T^{n}\right)$. In conjunction with (3.1.12), we can now assert that $u_{1}\left(z_{1}, z^{\prime}\right)$ is the Poisson integral of $u_{1}\left(a+\rho w_{1}, w^{\prime}\right)$ in $D_{1} \times U^{n-1}$. Consequently, $f_{1}^{\prime \prime} \in P\left(D_{1} \times U^{n-1}\right)$. A parallel argument shows that there is a disc $D_{2}$ containing $\lambda_{2}$ such that $f_{2}^{\prime \prime} \in P\left(D_{2} \times U^{n-1}\right)$.

For the next proposition consider the open intervals $J_{1}=(-1,1 / 2)$, $J_{2}=(-1 / 2,1)$, and $J=(-1,1)$. Let $K$ be an arbitrary bounded open interval. Define the rectangles $Q_{1}=J_{1}+i K, Q_{2}=J_{2}+i K$, $Q=J+i K$, and let $L=L_{2} \times L_{3} \times \cdots \times L_{n}$ be an arbitrary polyrectangle (open) in $C^{n-1}$.

Proposition 3.2. (Generalized Cartan lemma). If $g \in P\left(\left(Q_{1} \cap\right.\right.$ $\left.\left.Q_{2}\right) \times L\right)$, there exist $g_{1} \in P\left(Q_{1} \times L\right)$ and $g_{2} \in P\left(Q_{2} \times L\right)$ such that 
$g=g_{1}+g_{2}$ on $\left(Q_{1} \cap Q_{2}\right) \times L$.

Proof. Without loss of generality, assume that the rectangles $L_{m}$ are all equal. Let $\dot{\phi}$ be a conformal mapping from the disc $U$ onto $L_{m}$, and $\psi$ be a conformal mapping from $U$ to $Q_{1} \cap Q_{2}$. Extend $\psi$ to a homeomorphism between the closures $\bar{U}$ and $\overline{Q_{1} \cap Q_{2}}$. Let $\Lambda_{1}=\{-1 / 2+i y: y \in K\}, \Lambda_{2}=\{1 / 2+i y: y \in K\}$, and $\lambda_{1}, \lambda_{2}$ be the preimages of $\Lambda_{1}, \Lambda_{2}$ under $\psi$. Let $V_{1}, V_{2}$ be the domains constructed from $\lambda_{1}, \lambda_{2}$ as in (3.1). By the reflection principle, we can extend $\psi$ to a conformal mapping $\psi_{1}$ from $V_{1}$ onto the rectangle $S_{1}=$ $(-1 / 2,3 / 2)+i K$; i.e., given $|z|>1$ define $\psi_{1}(z)=1-\bar{\psi}(1 / \bar{z})$. Similarly, $\psi$ can be extended to a conformal mapping $\psi_{2}$ from $V_{2}$ onto $S_{2}=(-3 / 2,1 / 2)+i K$.

Define $\Phi: U^{n} \rightarrow\left(Q_{1} \cap Q_{2}\right) \times L$ by $\Phi\left(z_{1}, z_{2}, \cdots, z_{n}\right)=\left(\psi\left(z_{1}\right), \phi\left(z_{2}\right), \cdots\right.$, $\left.\phi\left(z_{n}\right)\right)$, and let $\Phi_{j}: V_{j} \times U^{n-1} \rightarrow S_{j} \times L$ be the extension of $\Phi$ obtained replacing $\psi$ by $\psi$, for $j=1,2$.

Suppose $g \in P\left(\left(Q_{1} \cap Q_{2}\right) \times L\right)$. Since the composition $f=g \circ \Phi$ is in $P\left(U^{n}\right)$, there exist functions $f_{1}, f_{2}$ satisfying the properties (a), (b), (c), and (d) of (3.1). If $g_{j}=f_{j} \circ \Phi_{j}$, the following can be verified:

(a') $g=g_{1}+g_{2}$ on $\left(Q_{1} \cap Q_{2}\right) \times L$,

(b') $g_{j} \in P\left(\left(Q_{1} \cap Q_{2}\right)\right) \times L$,

(c') $g_{j} \in P\left(\left(S_{j}-\overline{Q_{1} \cap Q_{2}}\right)\right) \times L$,

(d') $g_{j} \in P\left(\Phi_{j}\left(D_{j} \times U^{n-1}\right)\right)$, for $j=1,2$.

We claim that $g_{j} \in P\left(Q_{j} \times L\right)$.

The set $\psi_{1}\left(D_{1}\right)$ is the intersection of an open subset of $C$, that contains $\lambda_{1}$, with $S_{1}$, and $\Phi_{1}\left(D_{1} \times U^{n-1}\right)=\psi_{1}\left(D_{1}\right) \times L$. Consequently, we can find a relatively open polydomain $W_{\delta}$ in the closure $\overline{S_{1} \times L}$ such that $W_{\delta} \cap\left(S_{1} \times L\right)=\Phi_{1}\left(D_{1} \times U^{n-1}\right)$. It is also clear that there are relatively open polydomains $W_{\beta}$ and $W_{\gamma}$ in $\overline{Q_{1} \times L}$ such that $W_{\beta} \cap$ $\left(Q_{1} \times L\right)=\left(Q_{1} \cap Q_{2}\right) \times L$, and $W_{r} \cap\left(Q_{1} \times L\right)=\left(Q_{1}-\overline{Q_{1} \cap Q_{2}}\right) \times L$. Thus we have a covering $W_{\beta}, W_{\gamma}, W_{\delta}$ of $\overline{Q_{1} \times L}$ with the properties:

$\begin{array}{ll}\left(\mathrm{b}^{\prime \prime}\right) & g_{1} \in P\left(W_{\beta} \cap\left(Q_{1} \times L\right)\right), \\ \left(\mathrm{c}^{\prime \prime}\right) & g_{1} \in P\left(W_{\gamma} \cap\left(Q_{1} \times L\right)\right), \\ \left(\mathrm{d}^{\prime \prime}\right) & g_{1} \in P\left(W_{\delta} \cap\left(Q_{1} \times L\right)\right) .\end{array}$

The hypotheses of (2.1) are satisfied, so $g_{1} \in P\left(Q_{1} \times L\right)$. A parallel argument shows that $g_{2} \in P\left(Q_{2} \times L\right)$.

IV. The Čech cohomology of $\bar{U}^{n}$ with coefficient in $Q_{\text {. Our }}$ goal is that $H^{q}\left(\bar{U}^{n}, Q^{D}\right)=0$ for all integers $q \geqq 1$. The standard exact sequence $0 \rightarrow Z \rightarrow \mathscr{P} \stackrel{\exp 2 \pi i}{\longrightarrow} Q \rightarrow 0$ reduces this to proving $H^{q}\left(\bar{U}^{n}, \mathscr{P}\right)=0$. If $X$ is the cartesian product of $n$ bounded open rectangles in $\boldsymbol{C}$, we have analogous sheaves $\mathscr{P}$ and $\mathscr{Q}$ on $\bar{X}$, and the vanishing of the cohomology of $\bar{U}^{n}$ with coefficients in $\mathscr{P}^{\circ}$ 
is entirely equivalent to the corresponding result for $\bar{X}$. In the sequel, we work with $X$ instead of $U^{n}$. The reason for this preference is that it allows for the systematic partitioning into smaller polyrectangles used in (4.4).

Let $I=(-1,1)$. Define $R=I+i I$, and set $X=R^{n}$. Let $\mathscr{W}$ be the family of all cartesian products of open rectangles whose edges are parallel to the real and imaginary axes of $C$.

Fix an open covering $\mathscr{U} \subset \mathscr{W}$ of $\bar{X} . A q$-simplex $\sigma$ of $\mathscr{U}$ is a $q+1$-tuple $\left(U_{0}, U_{1}, \cdots, U_{q}\right)$ of sets in $\mathscr{C}$; its support $|\sigma|$ is the intersection $U_{0} \cap U_{1} \cap \cdots \cap U_{q}$. If $W \in \mathscr{W}$ is contained in $X$, define $C^{q}(\mathscr{C}(W), P)$ to be the group of all alternating functionals $\gamma(q-$ cochains) that assign to each $q$-simplex $\sigma$ of $\mathscr{C}$ a function $\gamma(\sigma)$ in $P(|\sigma| \cap W)$ (the zero function if the intersection is empty), and let $\delta: C^{q}(\mathscr{U}(W), P) \rightarrow C^{q+1}(\mathscr{U}(W), P)$ be the standard coboundary operator. The groups $C^{q}(\mathscr{U}(W), P)$ together with the homomorphisms $\delta$ form a cochain complex with cocycles $Z^{q}(\mathscr{U}(W), P)$, coboundaries $B^{q}(\mathscr{U}(W)$, $P)$, and cohomology groups $H^{q}(\mathscr{U}(W), P)$. It is an immediate consequence of (2.1) that $H^{0}(\mathscr{U}(W), P)$ equals $P(W)$. We define $H^{q}(\mathscr{C}(W), P)=0$ if $q<0$.

If $V$ is a polyrectangle in $\mathscr{W}^{-}$such that $V \subset W$, we have restriction homomorphisms $\rho_{V W}: C^{q}(\mathscr{U}(W), P) \rightarrow C^{q}(\mathscr{U}(V), P)$ which can easily be seen to commute with the coboundary operators. (If $\gamma \in$ $C^{q}(\mathscr{U}(W), P)$ and $\sigma$ is a $q$-simplex of $\mathscr{U}, \rho_{V W} \gamma(\sigma)$ is the restriction of the function $\gamma(\sigma)$ to $|\sigma| \cap V$.) When clear in the context, we shall denote $\rho_{V W} \gamma$ also by $\gamma$, and refer to it as the restriction of $\gamma$ to $\mathscr{U}(V)$.

For (4.1), (4.2), (4.3), let $I_{1}=(-1,1 / 2), I_{2}=(-1 / 2,1), R_{1}=I_{1}+$ $i I, R_{2}=I_{2}+i I$, and set $X_{1}^{1}=R_{1} \times R^{n-1}, X_{1}^{2}=R_{2} \times R^{n-1}$.

LEMMA 4.1. If $q \geqq 0$ and if $\gamma \in C^{q}\left(\mathscr{Q}\left(X_{1}^{1} \cap X_{1}^{2}\right), P\right)$, there exist $\gamma_{1} \in C^{q}\left(\mathscr{U}\left(X_{1}^{1}\right), P\right)$ and $\gamma_{2} \in C^{q}\left(\mathscr{U}\left(X_{1}^{2}\right), P\right)$ such that $\gamma=\gamma_{1}-\gamma_{2}$, with the appropriate restrictions to $\mathscr{U}\left(X_{1}^{1} \cap X_{1}^{2}\right)$.

Proof. We first observe that (3.2) remains valid if $J_{1}, J_{2}, J$ are arbitrary open intervals such that $J_{1} \cup J_{2}=J$, and such that either $J_{1} \subset J_{2}$, or $J_{2} \subset J_{1}$, or length $J_{1} \geqq 1 / 2$ length $J$, and length $J_{2} \geqq 1 / 2$ length $J$. If $\sigma$ is a $q$-simplex of $\mathscr{C}$, the polyrectangles $|\sigma| \cap X_{1}^{1}$, $|\sigma| \cap X_{1}^{2},|\sigma| \cap X_{1}^{1} \cap X_{1}^{2}$, will satisfy the modified hypotheses of (3.2); they can be taken as the polyrectangles $Q_{1} \times L, Q_{2} \times L$, and $\left(Q_{1} \cap\right.$ $\left.Q_{2}\right) \times L$ of (3.2).

Let $\gamma \in C^{q}\left(\mathscr{C}\left(X_{1}^{1} \cap X_{1}^{2}\right), P\right)$ and let $\sigma$ be a $q$-simplex of $\mathscr{W}$. Since $\gamma(\sigma) \in P\left(|\sigma| \cap X_{1}^{1} \cap X_{1}^{2}\right)$, we can decompose it as a difference $\gamma_{1}(\sigma)-$ $\gamma_{2}(\sigma)$ of functions $\gamma_{j}(\sigma) \in P\left(|\sigma| \cap X_{1}^{j}\right)$. Repeating this for each $q$ simplex we construct $\gamma_{1} \in C^{q}\left(\mathscr{C}\left(X_{1}^{1}\right), P\right), \gamma_{2} \in C^{q}\left(\mathscr{U}\left(X_{1}^{2}\right), P\right)$ such that 
$\gamma_{1}-\gamma_{2}=\gamma$

Lemma 4.2. Let $q \geqq 0$. If $\gamma_{1} \in C^{q}\left(\mathscr{U}\left(X_{1}^{1}\right), P\right)$ and $\gamma_{2} \in C^{q}\left(\mathscr{U}\left(X_{1}^{2}\right), P\right)$ have identical restrictions to $\mathscr{Q}\left(X_{1}^{1} \cap X_{1}^{2}\right)$, then $\gamma_{1}$ and $\gamma_{2}$ must be the restrictions to $\mathscr{U}\left(X_{1}^{1}\right)$ and $\mathscr{U}\left(X_{1}^{2}\right)$, respectively, of some $\gamma \in$ $C^{q}(\mathscr{U}(X), P)$.

Proof. Let $\sigma$ be a $q$-simplex of $\mathscr{U}$. Then $\gamma_{1}(\sigma) \in P\left(|\sigma| \cap X_{1}^{1}\right)$, $\gamma_{2}(\sigma) \in P\left(|\sigma| \cap X_{1}^{2}\right)$, and $\gamma_{1}(\sigma)$ agrees with $\gamma_{2}(\sigma)$ on the intersection $|\sigma| \cap X_{1}^{1} \cap X_{1}^{2}$. Let $\gamma(\sigma)$ be the analytic continuation of $\gamma_{1}(\sigma)$ given by $\gamma_{2}(\sigma)$. It follows from (2.1) that $\gamma(\sigma) \in P(|\sigma| \cap X)$. Repeating this procedure for each $\sigma$, we define $\gamma \in C^{q}(\mathscr{U}(X), P)$ with the requirements of the lemma.

DEFINITION 4.3. For each integer $q \geqq 0$, we construct the sequence of homomorphisms $0 \rightarrow C^{q}\left(\mathscr{U}\left(X_{1}^{1} \cup X_{1}^{2}\right), P\right) \stackrel{\phi}{\rightarrow} C^{q}\left(\mathscr{U}\left(X_{1}^{1}, P\right) \oplus\right.$ $C^{q}\left(\mathscr{C}\left(X_{1}^{2}\right), P\right) \stackrel{\varphi}{\rightarrow} C^{q}\left(\mathscr{\mathscr { C }}\left(X_{1}^{1} \cap X_{1}^{2}\right), P\right) \rightarrow 0$, where $\phi(\gamma)=(\gamma, \gamma)$ and $\psi\left(\gamma_{1}\right.$, $\left.\left.\gamma_{2}\right)\right)=\gamma_{1}-\gamma_{2}$ (with obvious restrictions). Lemmas (4.1), (4.2) assert that it is an exact sequence. It can be verified that the homomorphisms $\phi, \psi$ commute with the coboundary operator $\delta$. Consequently, the above is a short exact sequence of the cochain complexes $\left\{C^{q}\left(\mathscr{U}\left(X_{1}^{1} \cup X_{1}^{2}\right), P\right), \delta\right\},\left\{C^{q}\left(\mathscr{U}\left(X_{1}^{1}\right), P\right) \oplus C^{q}\left(\mathscr{U}\left(X_{1}^{2}\right), P\right), \delta \oplus \delta\right\},\left\{C^{q}\left(\mathscr{U}\left(X_{1}^{1} \cap\right.\right.\right.$ $\left.\left.\left.X_{1}^{2}\right), P\right), \delta\right\}$. As is well known ([1, Th. 3.7, p. 128]), there is an associated long exact sequence

$$
\begin{aligned}
0 \longrightarrow & \cdots \longrightarrow H^{q-1}\left(\mathscr{U}\left(X_{1}^{1} \cap X_{1}^{2}\right), P\right) \\
& \stackrel{\delta^{*}}{\longrightarrow} H^{q}\left(\mathscr{C}\left(X_{1}^{1} \cup X_{1}^{2}\right), P\right) \\
& \stackrel{\phi^{*}}{\longrightarrow} H^{q}\left(\mathscr{U}\left(X_{1}^{1}\right), P\right) \oplus H^{q}\left(\mathscr{U}\left(X_{1}^{2}\right), P\right) \\
& \stackrel{\varphi^{*}}{\longrightarrow} H^{q}\left(\mathscr{\mathscr { C }}\left(X_{1}^{1} \cap X_{1}^{2}\right), P\right) \stackrel{\delta^{*}}{\longrightarrow} \cdots .
\end{aligned}
$$

Since, by (3.2), $0 \rightarrow P\left(X_{1}^{1} \cup X_{1}^{2}\right) \stackrel{\phi}{\rightarrow} P\left(X_{1}^{1}\right) \oplus P\left(X_{1}^{2}\right) \stackrel{\phi}{\rightarrow} P\left(X_{1}^{1} \cap X_{1}^{2}\right) \rightarrow 0$ is exact, we can assume that in (4.3.1) the first term following zero is $H^{1}\left(\mathscr{U}\left(X_{1}^{1} \cup X_{1}^{2}\right), P\right)$.

Proposition 4.4. For any polyrectangle $X$ in $\mathscr{W}$, for any covering $\mathscr{U} \subset \mathscr{W}$ of $\bar{X}$, and for any integer $q \geqq 1$, the cohomology groups $H^{q}(\mathscr{U}(X), P)$ are trivial.

Proof. We argue by induction. Suppose that either $q=1$, or that $q>1$ and the proposition is true for all positive integers $\leqq$ $q-1$. Let $X$ be a member of $\mathscr{W}$; assume without loss of generality that $X=X_{1}^{1} \cup X_{1}^{2}$ is the polyrectangle of (4.3.1). 
If $q>1$, the inductive hypothesis, applied to $X_{1}^{1} \cap X_{1}^{2}$, implies $H^{q-1}\left(\mathscr{C}\left(X_{1}^{1} \cap X_{1}^{2}\right), P\right)=0$. Hence, the homomorphism

$$
H^{q}\left(\mathscr{\mathscr { C }}\left(X_{1}^{1} \cup X_{1}^{2}\right), P\right) \stackrel{\phi^{*}}{\longrightarrow} H^{q}\left(\mathscr{U}\left(X_{1}^{1}\right), P\right) \oplus H^{q}\left(\mathscr{U}\left(X_{1}^{2}\right), P\right)
$$

is one-one for all $q \geqq 1$ (the case $q=1$ is trivial).

Suppose that $H^{q}(\mathscr{U}(X), P) \neq 0$, and let $\zeta$ be a cocycle in $Z^{q}(\mathscr{U}(X), P)$ that does not cobound. Since $\phi^{*}$ in (4.4.1) is one-one, the restrictions of $\zeta$ to $\mathscr{U}\left(X_{1}^{1}\right)$, and to $\mathscr{U}\left(X_{1}^{2}\right)$, cannot both cobound. Let $X_{1}^{k_{1}}$ be the polyrectangle on which $\zeta$ fails to cobound. The procedure that led to (4.3.1) can be repeated for $X_{1}^{k_{1}}$, a subdivision $X_{1}^{k_{1}}=X_{2}^{1} \cup X_{2}^{2}$, and the same covering $\mathscr{U}$. As before, if we apply the inductive hypothesis to $X_{2}^{1} \cap X_{2}^{2}$, the homomorphism

$$
H^{q}\left(\mathscr{C}\left(X_{2}^{1} \cup X_{2}^{2}\right), P\right) \stackrel{\phi^{*}}{\longrightarrow} H^{q}\left(\mathscr{U}\left(X_{2}^{1}\right), P\right) \oplus H^{q}\left(\mathscr{C}\left(X_{2}^{2}\right), P\right)
$$

will also be one-one. Iterating this procedure, proceeding cyclicly through the real and imaginary coordinates of $\boldsymbol{C}^{n}$, we obtain a nested sequence $X_{1}^{k_{1}} \supset X_{2}^{k_{2}} \supset \cdots \supset X_{m}^{k_{m}} \supset \cdots$ of polyrectangles with diameters eventually decreasing to zero, on none of which the cocycle induced by $\zeta$ cobounds. This leads to a contradiction: $\mathscr{U}=\left\{U_{\alpha}\right\}$ is an open covering of $\bar{X}$, so for some integer $m$ and some $U_{\alpha}$ in the covering, we will have $X_{m}^{k_{m}} \subset U_{\alpha}$; if $m$ is so chosen, the restriction of $\zeta$ to $\mathscr{U}\left(X_{m}^{k_{m}}\right)$ trivially cobounds, i.e., if $\gamma$ is defined by $\gamma\left(U_{0}, \cdots, U_{q-1}\right)=$ $\zeta\left(U_{\alpha}, U_{0}, \cdots, U_{q-1}\right)$, then $\delta \gamma=\zeta$.

CoRollary 4.5. Let $X$ be a polyrectangle in $C^{n}$ and $U^{n}$ be the unit polydisc in $\boldsymbol{C}^{n}$. Then, for all integers $q \geqq 1$,

(a) $H^{q}(\bar{X}, \mathscr{P})=0$,

(b) $H^{q}\left(\bar{U}^{n}, \mathscr{P}\right)=0$,

(c) $H^{q}\left(\bar{U}^{n}, \mathscr{Q}\right)=0$.

Proof. As was noted earlier, (b) and (c) are direct consequences of (a).

To prove (a) it suffices to show that $H^{q}(\mathscr{Y}, \mathscr{P})=0$ for any covering $\mathscr{V} \subset \mathscr{W}$ of $\bar{X}$ (since such coverings are cofinal in the class of all open coverings of $\bar{X}$ ). Choose such a covering $\mathscr{V}$, and let $\mathscr{U} \subset \mathscr{W}$ be a refinement of $\mathscr{V}$ such that the closure of each member $U$ of $\mathscr{C}$ is contained in some polyrectangle $\mu U$ of $\mathscr{Y}$. Let $\sigma=$ $\left(U_{0}, U_{1}, \cdots, U_{q}\right)$ be a simplex of $\mathscr{\mathscr { C }}$ and let $\mu \sigma=\left(\mu U_{0}, \mu U_{1}, \cdots, \mu U_{q}\right)$ be the corresponding simplex of $\mathscr{Y}$. Recall that a section $\gamma \in$ $\Gamma(|\mu \sigma|, \mathscr{P})$ can be naturally identified with a holomorphic function $f_{r}$ in $|\mu \sigma| \cap X$, and that the restriction of $f_{\gamma}$ to $|\sigma| \cap X$ will be in $P(|\sigma| \cap X)$. With this in mind, we construct, for each integer $q \geqq 0$, a one-one homomorphism 


$$
C^{q}(\mathscr{T}, \mathscr{P}) \stackrel{\mu^{*}}{\longrightarrow} C^{q}(\mathscr{U}(X), P)
$$

defined by letting $\mu^{*}(\gamma)(\sigma)$ be the function $f_{\gamma}$ restricted to $|\sigma| \cap X$. A straightforward calculation shows that $\mu^{*}$ commutes with the coboundary operators; consequently it induces a one-one homomorphism

$$
H^{q}(\mathscr{Y}, \mathscr{P}) \stackrel{\mu^{*}}{\longrightarrow} H^{q}(\mathscr{U}(X), P) \text {. }
$$

It follows, by (4.4), that $H^{q}(\mathscr{V}, \mathscr{P})=0$, which completes the proof.

V. Remarks. In [7] Stout proved that the multiplicative Cousin problem with bounded data can be solved in the polydisc $U^{n}$. If, as in [6], we let $\mathscr{C}$ be the sheaf of germs of locally bounded holomorphic functions, and $\mathscr{E}$ be the sheaf of multiplicative groups of invertible elements of $\mathscr{Z}$, this is equivalent to the assertion that $H^{1}\left(\bar{U}^{n}, \mathscr{E}\right)$ is trivial. If we apply the methods of $\S I V$ to the sheaf $\mathscr{P}_{b}$ (defined in [6]) of locally bounded pluriharmonic functions on $\bar{U}^{n}$, it follows that $H^{q}\left(\bar{U}^{n}, \mathscr{E}\right)=0$ for all $q>1$, as well.

The methods used for the study of the sheaf $\mathscr{P}$ can be also applied to obtain similar results for the sheaves $\mathscr{H}^{p}$, induced by assigning to each relatively open polydomain $W \subset \bar{U}^{n}$ the Hardy space $\mathscr{H}^{p}\left(W \cap U^{n}\right)$. If $p>1$, Lemma 3.1 holds word for word if everywhere we replace the letter $P$ by the symbol $\mathscr{C}^{p}$; it then can be proven, as was done for the sheaf $\mathscr{P}$, that $H^{q}\left(U^{n}, \mathscr{H}^{p}\right)=0$ for all $q \geqq 1$. A simpler procedure, however, is to show that the sheaves $\mathscr{H}^{p}$ correspond to a particular case of the boundary conditions studied by Nagel in [4].

Finally, we mention that as a consequence of $H^{1}\left(\bar{U}^{n}, \mathscr{Q}\right)=0$, it is possible to solve the multiplicative Cousin problem with $N^{*}$-data in $U^{n}$ (in [8], the corresponding problem for the Nevanlinna class $N$ was shown to be solvable). By standard arguments (such as in [3, Cor. 2, p. 47]) it can be shown that $H^{1}(\mathscr{U}, \mathbb{Q})=0$ for any covering $\mathscr{U}=\left\{U_{\alpha}\right\}$ of $\bar{U}^{n}$. If $\mathscr{\mathscr { C }}$ consists of relatively open polydomains, and if for each $\alpha$ we are given $f_{\alpha} \in N^{*}\left(U_{\alpha} \cap U^{n}\right)$ such that $f_{\alpha} f_{\beta}^{-1}$ is an outer function in the intersection $U_{\alpha} \cap U_{\beta} \cap U^{n}$ ( a cocycle in $Z^{1}(\mathscr{U}, \mathscr{Q})$ ), there must exist $F \in N^{*}\left(U^{n}\right)$ with the property that $F f_{\alpha}^{-1}$ is an outer function in $U_{\alpha} \cap U^{n}$ for each $\alpha$.

\section{REFERENCES}

1. S. Eilenberg and N. Steenrod, Foundations of algebraic topology, Princeton Mathematical Series, Princeton University Press, Princeton, (1952).

2. P. M. Gauthier and W. Hengartner, Local harmonic majorants of functions subharmonic in the unit disc, J. Analyse Math., 26 (1973), 405-412.

3. R. C. Gunning, Lectures on Riemann surfaces, Princeton Mathematical Notes, Princeton University Press, Princeton, (1966). 
4. A. Nagel, Cohomology of sheaves of holomorphic functions satisfying boundary conditions on product domains, Trans. Amer. Math. Soc., 172 (1972), 133-141.

5. W. Rudin, Function Theory in Polydiscs, Mathematics Lecture Notes Series, W. A. Benjamin, Inc., New York, (1969).

6. E. L. Stout, On the multiplicative Cousin problem with bounded data, Annali della Scuola Normale Superiore di Pisa, Vol XXVII, Fasc., 1 (1973), 1-17.

7. - The second Cousin problem with bounded data, Pacific J. Math., 26 (1968), 379-387.

8. S. E. Zarantonello, The multiplicative Cousin problem and a zero set problem for the Nevanlinna class in the polydisc, Trans. Amer. Math. Soc., 200 (1974), 291-313.

Received July 7, 1977 and in revised form October 14, 1977.

UNIVERSITY OF FLORIDA

Gainesville, FL 32611 



\section{PACIFIC JOURNAL OF MATHEMATICS}

\section{EDITORS}

RICHARD ARENS (Managing Editor)

University of California

Los Angeles, California 90024

C. W. CURTIS

University of Oregon

Eugene, OR 97403

C. C. MOORE

University of California

Berkeley, CA 94720

\section{J. DUGUNDJI}

Department of Mathematics University of Southern California Los Angeles, California 90007

R. Finn aNd J. Milgram Stanford University Stanford, California 94305

\section{ASSOCIATE EDITORS}

E. F. BeCKenbaCH

B. H. NeumanN

F. WOLF

K. YosHIDA

\section{SUPPORTING INSTITUTIONS}

UNIVERSITY OF BRITISH COLUMBIA CALIFORNIA INSTITUTE OF TECHNOLOGY UNIVERSITY OF CALIFORNIA MONTANA STATE UNIVERSITY UNIVERSITY OF NEVADA, RENO NEW MEXICO STATE UNIVERSITY OREGON STATE UNIVERSITY UNIVERSITY OF OREGON
UNIVERSITY OF SOUTHERN CALIFORNIA STANFORD UNIVERSITY UNIVERSITY OF HAWAII UNIVERSITY OF TOKYO UNIVERSITY OF UTAH WASHINGTON STATE UNIVERSITY UNIVERSITY OF WASHINGTON 


\section{Pacific Journal of Mathematics}

\section{Vol. 76, No. $1 \quad$ November, 1978}

Ata Nuri Al-Hussaini, Potential operators and equimeasurability ......... 1

Tim Anderson and Erwin Kleinfeld, Semisimple nil algebras of type $\delta . \ldots .99$

Stephen LaVern Campbell, Linear operators for which $T^{*} T$ and $T+T^{*}$

commute. III ......................................

Robert Jay Daverman, Special approximations to embeddings of codimension one spheres...............................

Donald M. Davis, Connective coverings of $\mathrm{BO}$ and immersions of projective

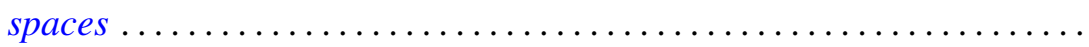

V. L. (Vagn Lundsgaard) Hansen, The homotopy type of the space of maps of

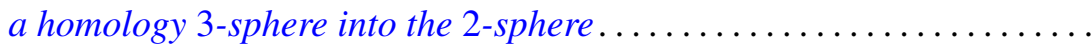

James Victor Herod, A product integral representation for the generalized

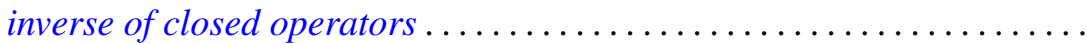

A. A. Iskander, Definability in the lattice of ring varieties ..............

Russell Allan Johnson, Existence of a strong lifting commuting with a

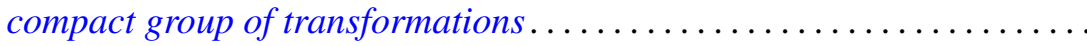

Heikki J. K. Junnila, Neighbornets...................... 83

Klaus Kalb, On the expansion in joint generalized eigenvectors . ......... 109

F. J. Martinelli, Construction of generalized normal numbers . . . . . . . . . 117

Edward O'Neill, On Massey products ....................... 123

Vern Ival Paulsen, Continuous canonical forms for matrices under unitary

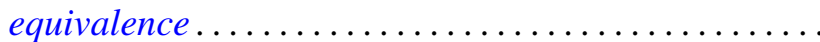

Justin Peters and Terje Sund, Automorphisms of locally compact groups . . . 143

Duane Randall, Tangent frame fields on spin manifolds . . . .

Jeffrey Brian Remmel, Realizing partial orderings by classes of co-simple sets . . . .

J. Hyam Rubinstein, One-sided Heegaard splittings of 3-manifolds ...

Donald Charles Rung, Meier type theorems for general boundary approach

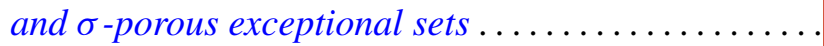

Ryōtarō Satō, Positive operators and the ergodic theorem

Ira H. Shavel, A class of algebraic surfaces of general type constructed from

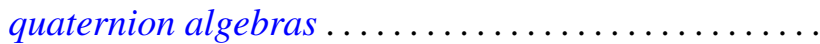

Patrick F. Smith, Decomposing modules into projectives and injectives ....

Sergio Eduardo Zarantonello, The sheaf of outer functions in the polydisc... 